Noman 2016, 34(1), 33-43

Revista de Psicologia, Ciències de l'Educació i de l'Esport

ISSN: $1138-3194$

Copyright (c) 2016

www.revistaaloma.net

\title{
La innovación como competencia docente en la universidad: Innovación orientada a la mejora de aprendizaje
}

\author{
Teresa Pagés ${ }^{1}$, Carme Hernández ${ }^{2}$, Ana Rosa Abadía $^{3}$, Concha Bueno ${ }^{3}$, Isabel Ubieto-Artur ${ }^{3}$, \\ Dolors Márquez ${ }^{4}$, Sarai Sabaté ${ }^{4} \&$ Helga Jorba ${ }^{1}$ \\ 'Universitat de Barcelona, ${ }^{2}$ Universitat Pompeu Fabra, ${ }^{3}$ Universidad de Zaragoza, \\ ${ }^{4}$ Universitat Autònoma de Barcelona
}

Recibido: 19-12-2015

Aceptado: 4-3-2016

La innovación como competencia docente en la universidad: Innovación orientada a la mejora de aprendizaje

Resumen. En este trabajo valoramos la competencia de innovación docente del profesorado universitario como medio para mejorar la calidad de la docencia y conseguir un mejor aprendizaje de los estudiantes. Esta competencia es una de las seis definidas como relevantes, y considerada como una competencia significativa por el Grupo Interuniversitario de Formación Docente (GIFD), y por el grupo de expertos externos. La validación de estas competencias se realizó a partir de los datos recogidos en encuestas dirigidas al profesorado y a los estudiantes de las universidades españolas. La doble visión proporcionada por los profesores y los estudiantes sirvió para redefinir mejor las dimensiones de cada competencia y los indicadores correspondientes a cada dimensión. Estos indicadores se organizaron por niveles, y se propusieron evidencias para poder valorar el nivel competencial del profesorado. Esta valoración puede ayudar a promover la mejora profesional del profesorado a través de programas de formación docente basado en competencias, redundando en una mejora de su actuación en el aula, y por tanto, del aprendizaje de los estudiantes. Puede servir, además, como sistema de soporte para evaluar la calidad de la docencia en los procesos de acreditación del profesorado de las agencias de calidad universitaria, autonómica y estatal.

Palabras clave: Competencias docentes; innovación docente; desarrollo competencial; formación profesorado universitario

Innovation as a university teaching competency: innovation aimed at improvements in learning

Summary. This paper is an assessment of university professors' competency in innovation as a means to improve the quality of their teaching and enhance their students' learning process. This competency is one of the six that were identified as important and labelled as significant by the Grupo Interuniversitario de Formación Docente (GIFD) and the group of external experts. The competencies were validated using data collected via surveys of professors and students at Spanish universities. The dual perspective provided by faculty and students allowed for a better redefinition of the dimensions of each of the competencies and of the corresponding indicators for each of these dimensions. These indicators were organised into a level-based rating system, and proposals were made as to the data to be used to assess the competency level of faculty members. This assessment could help spur professional improvement among the faculty via competency-based teacher training programs, with ultimate repercussions in the form of improved classroom performance by teachers and a resulting improvement in students' learning processes. The assessment could also be used as an additional tool to evaluate teaching quality in faculty certification processes carried out by university, regional and national quality assurance agencies.

Keywords: teaching competencies; innovation in teaching; competency development; university faculty training

Correspondencia

Teresa Pagès

Universitat de Barcelona

Departament de Biologia Cel·lular, Fisiologia i Immunologia

Facultat de Biologia

Av. Diagonal, 643

08028 Barcelona

email: tpages@ub.edu

http://orcid.org/0000-0003-1083-5514 


\section{Introducción}

La construcción del Espacio Europeo de Educación Superior (EEES), los cambios sociales, la extensión de la democratización y su aplicación a los sistemas formativos han supuesto un cambio en el rol de los docentes (Rodríguez-Gómez \& Gairín Sallán, 2015, p. 75) $y$, por tanto, cambios en la manera de enfocar la formación del profesorado universitario para adecuarse al nuevo paradigma que potencia el aprendizaje del estudiante dentro del binomio enseñanza-aprendizaje característico del nuevo modelo formativo. El paso de un modelo de formación a otro, nada inmediato ni simple, se realiza a través de la innovación educativa, con la introducción de cambios progresivos para mejorar el aprendizaje. En la Universidad, la innovación educativa es una necesidad para dar respuesta a los nuevos requerimientos sociales, que implica un profundo cambio de mentalidad de los actores del proceso, tanto de docentes como de discentes, y sobre principios básicos que le confieren eficacia y validez.

En el entorno socioprofesional, se valora que los titulados universitarios tengan la capacidad para innovar (González \& Wagenaar, 2003, 2006), pues constituye una de las competencias transversales más demandadas en cualquier titulado universitario, por lo que a su vez tiene que contemplarse en el perfil docente universitario. Las instituciones educativas «como estructuras de progreso que promueven el cambio y asumen su compromiso social» (Rodríguez-Gómez \& Gairín Sallán, 2015, p. 75) han de fomentar la competencia de innovación con una nueva perspectiva desde el punto de vista organizativo: «la competencia asume una nueva perspectiva a la luz de la concepción de las organizaciones como elementos dinamizadores de la sociedad y preparadas para el cambio constante, capaces de adelantarse y de buscar alternativas» (Gairín, 2011).

La innovación es indispensable para que la educación progrese pero, para ello, es necesario definir y concretar cuáles son sus características para que pueda considerarse la innovación como innovación educativa (Parcerisa, Bascos, Calafell, Comas \& Noguera, 2011).

Actualmente se entiende el proceso educativo innovador desde la perspectiva de la calidad de la educación y de la estructura organizativa, más que de la calidad de la formación en el aula (Rodríguez-Gómez \& Gairín Sallán, 2015, p. 87). El concepto de innovación debería revisarse para incorporar aspectos relacionados con el aprendizaje organizativo, que facilitaran la innovación y la creatividad, tanto a nivel individual como grupal (Rodríguez-Gómez \& Gairín Sallán, 2015, p. 80-83). El desarrollo competencial innovador se valora como necesario, pero requiere un marco de referencia específico, como son los programas formativos, dentro de la estructura organizativa de las universidades.

En este contexto, resulta necesario avanzar hacia un enfoque institucional de la innovación que fomente buenas prácticas que faciliten al profesorado, agente del cambio, la promoción de acciones de mejora curricular y de efectividad en el aula (Biggs, 2003; Bain, 2006;
Barnett, 2011). Una de las principales cuestiones que se plantea actualmente es garantizar la sostenibilidad de la innovación docente. Los cambios sostenibles requieren de enfoques que abarquen tanto el desarrollo individual de los docentes como el desarrollo organizativo de las estructuras y de la cultura universitaria (Euler, 2015). Según Euler (2015, p. 151) «¿cómo pueden los cambios en la docencia iniciarse y convertirse en efectivos de manera sostenible? La discusión persigue la hipótesis de que la expansión de las actividades de formación de los profesores universitarios es esencial para desencadenar cambios en la enseñanza y el aprendizaje en las universidades con probabilidad de tener un efecto sostenible. Esta perspectiva conduce a un marco de referencia que se resume en la noción de 'desarrollo universitario en el ámbito de la enseñanza y el aprendizaje'. Este término representa el conjunto de todas las actividades en las universidades que pueden crear cambios específicos en la enseñanza y el aprendizaje.»

La calidad de la enseñanza universitaria ha de tender a la calidad del aprendizaje (Zabalza, 2013) y de los recursos personales promovidos por la institución, entre ellos la formación y los proyectos de innovación, como elementos clave de la calidad institucional (Rodríguez Conde, Olmos Migueláñez, Ortega Mohedano, Torrijos Fincias \& Hernández Garzón, 2014), lo que pone de relieve la necesidad del apoyo institucional a los proyectos de innovación y a la formación docente, así como a las acciones que inciden en el desarrollo de la competencia de innovación educativa. Uno de los retos que, en general, suscita más interés es el relacionado con la innovación metodológica docente (Parcerisa et al., 2011), orientada a implicar al alumnado, a organizar el espacio y el tiempo, a evaluar aprendizajes, y a atender la diversidad en el aula. Llena y Parcerisa (2008, p. 20) consideran que la calidad de la acción educativa "consiste en planificar, proporcionar y evaluar la ayuda educativa óptima para cada educando en cada situación o contexto específico».

Todas estas premisas enlazan con los objetivos llevados a cabo por el Grupo Interuniversitario de Formación Docente (GIFD) en el marco del proyecto Estudios y Análisis (EA 2010-0099), que analizaron las competencias docentes en el contexto social y profesional actual, identificando y definiendo qué competencias y qué indicadores se consideraban más relevantes para la función docente, desde el punto de vista del profesorado de las universidades públicas catalanas (Torra, 2011; Torra et al. 2012; Triadó, Estebanell, Márquez \& Del Corral, 2014). Esta investigación se completó a través de otro proyecto (REDU2012), en el que se amplió la muestra del profesorado al resto de universidades españolas y se recogió la opinión de los estudiantes con el fin de revisar y validar, en su caso, las competencias y los indicadores establecidos (Pagés, 2014; Abadía et al., 2015).

Las seis competencias definidas inicialmente por el grupo GIFD fueron la interpersonal, la metodológica, la comunicativa, la de planificación y gestión docente, la de trabajo en equipo y la de innovación docente. 
Tabla 1. Muestra de la encuesta del profesorado y de los estudiantes de grado / licenciatura y máster

\begin{tabular}{|c|c|c|c|c|c|c|}
\hline & Muestra total & $\begin{array}{l}\text { Muestra mínima } \\
\text { requerida* }^{*}\end{array}$ & $\begin{array}{l}\text { Muestra obtenida } \\
\text { (número respuestas) }\end{array}$ & \% Participación & $\begin{array}{l}\text { Muestra final } \\
\text { (encuestas válidas) }\end{array}$ & Error muestral \\
\hline Profesorado & 112.154 & 383 & 7.553 & $6.73 \%$ & 5.139 & $1.3 \%$ \\
\hline $\begin{array}{l}\text { Estudiantes grado o } \\
\text { licenciatura }\end{array}$ & 370.744 & 5.629 & 10.324 & $2.78 \%$ & 10.302 & $1 \%$ \\
\hline Estudiantes máster & 26.401 & 4.505 & 1.701 & $6.44 \%$ & 1.700 & $2.3 \%$ \\
\hline
\end{tabular}

Muestra total de profesorado del periodo 2011-2012a. Muestra total de estudiantes del periodo 2010-2011 .

(*) Muestra mínima calculada para cada universidad asegurando un error del 5\%, con una confianza del 95\% y una homogeneidad del 50\%.

a Datos del Sistema Integrado de Información Universitaria de la web del Ministerio de Educación, Cultura y Deporte. No incluye al profesorado de la Universidad Internacional Menéndez Pelayo (UIMP).

${ }^{\mathrm{b}}$ Datos obtenidos del Sistema Integrado de Información Universitaria de la web del Ministerio de Educación, Cultura y Deporte. Solo se incluye el estudiantado de las universidades que participan en el proyecto.

En este artículo nos centramos en la competencia de innovación docente, no como objetivo en sí misma, sino entendida desde el punto de vista de la capacidad para crear y aplicar nuevos conocimientos, perspectivas, metodologías y recursos en las diferentes dimensiones de la actividad docente, orientadas a la mejora de la calidad del proceso de enseñanza-aprendizaje, que entronca con la definición generalizada de la innovación educativa.

El objetivo de este estudio es contrastar la perspectiva docente y discente en relación con la competencia de innovación docente, para orientar el diseño de la formación docente y ayudar a que el profesorado pueda aplicar actividades de mejora e innovación en el aula para mejorar los resultados de aprendizaje de sus alumnos.

\section{Método}

\section{Participantes}

En el proyecto se han implicado 15 universidades. Las encuestas se distribuyeron entre el profesorado y los estudiantes de 55 universidades de 17 comunidades autónomas. El número de participantes, tanto de profesorado como de estudiantes, así como la muestra final utilizada para el análisis se especifica en la tabla 1. Los datos de PDI corresponden al periodo 2011-2012, ${ }^{1}$ y los de los estudiantes, al 2010-2011. ${ }^{2}$

Dadas las características particulares del colectivo de estudiantes de grado, respecto al de posgrado, y con el objeto de obtener la máxima representatividad y fiabilidad de los resultados obtenidos, se ha tratado a los estudiantes de grado y de máster como dos poblaciones separadas y, por tanto, se han considerado dos muestras.

\section{Material}

Para la recogida de datos del profesorado sobre las competencias docentes, se utilizó la encuesta validada por el grupo GIFD (Estudios y Análisis (EA2010-0099)

\footnotetext{
${ }^{1}$ Datos del Sistema Integrado de Información Universitaria de la web del Ministerio de Educación, Cultura y Deporte. No incluye al profesorado de la Universidad Internacional Menéndez Pelayo (UIMP).

${ }^{2}$ Datos obtenidos del Sistema Integrado de Información Universitaria de la web del Ministerio de Educación, Cultura y Deporte. Solo se incluye el estudiantado de las universidades que participan en el proyecto.
}

(Torra, 2011, p. 183-196). Para los estudiantes se elaboró y validó una nueva encuesta, más corta y accesible (Pagés, 2014, p. 164-168).

Para la encuesta para el profesorado se utilizó la plataforma Encuestafacil.com, que permite obtener copias de seguridad de manera regular e informes de participación detallados para el posterior análisis estadístico. La encuesta constaba de tres partes bien diferenciadas: a) perfil sociodemográfico del encuestado; b) cuestiones relacionadas con la importancia de las competencias o de los indicadores que las conforman (escala 1-4); c) priorización de las competencias.

Se incluyó un cuarto apartado, optativo, con un formato de pregunta abierta, se dejaba la posibilidad de incluir sugerencias y aportaciones.

La competencia de innovación docente estaba definida como: crear y aplicar nuevos conocimientos, perspectivas, metodologías y recursos en las diferentes dimensiones de la actividad docente, orientados a la mejora de la calidad del proceso de enseñanza- aprendizaje (Torra et al., 2012)

Los indicadores definidos son los siguientes:

- CDI1. Analizar el contexto de enseñanza-aprendizaje para identificar las necesidades de mejora y aplicar estrategias y/o recursos innovadores.

- CDI2. Reflexionar e investigar sobre los procesos de enseñanza-aprendizaje para buscar nuevas estrategias que permitan mejorarlos.

- CDI3. Definir un objetivo preciso de la innovación que se pretende llevar a cabo.

- CDI4. Adaptar las innovaciones a las características y particularidades de cada contexto.

- CDI5. Introducir innovaciones que tengan como objetivo una mejora del proceso de enseñanzaaprendizaje.

- CDI6. Participar activamente en proyectos y experiencias de innovación docente.

- CDI7. Evaluar y transferir resultados y experiencias de innovación al propio contexto de enseñanzaaprendizaje orientados a la mejora de la calidad docente.

La encuesta para los estudiantes se implementó con la herramienta JotForm (http://www.jotform.com), que permite crear formularios en línea optimizados para dispositivos móviles, y recoger la opinión de forma anónima.

Los ítems de la encuesta fueron redactados a partir de los componentes de las competencias definidas para 
Tabla 2. Equivalencia entre los ítems de la encuesta de los estudiantes y las competencias docentes del profesorado

\begin{tabular}{ll}
\hline Ítems & $\begin{array}{l}\text { Competencia } \\
\text { equivalente }\end{array}$ \\
\hline $\begin{array}{l}\text { 1. Estimular la reflexión, la crítica y la autocrítica } \\
\text { 2. Fomentar un clima de confianza y de tolerancia en el }\end{array}$ & CI \\
aula & CI \\
$\begin{array}{l}\text { 3. Motivar al alumnado en su proceso de aprendizaje } \\
\text { 4. Utilizar métodos de enseñanza-aprendizaje } \\
\text { coherentes con los objetivos de la asignatura }\end{array}$ & CI \\
$\begin{array}{l}\text { 5. Utilizar procedimientos de evaluación coherentes } \\
\text { con los objetivos de la asignatura }\end{array}$ & CM \\
$\begin{array}{l}\text { 6. Fomentar la participación y el trabajo colaborativo } \\
\text { 7. Proporcionar feedback sobre el proceso de } \\
\text { aprendizaje y favorecer su reorientación }\end{array}$ & CM \\
$\begin{array}{l}\text { 8. Explicar de forma clara los contenidos de la } \\
\text { asignatura }\end{array}$ & CC \\
$\begin{array}{l}\text { 9. Escuchar a los estudiantes (promover el diálogo) } \\
\text { 10. Informar de la planificación de la asignatura }\end{array}$ & CC \\
$\begin{array}{l}\text { 11. Destacar los contenidos más relevantes en la } \\
\text { titulación y la profesión }\end{array}$ & CPGD \\
$\begin{array}{l}\text { 12. Especificar claramente los criterios y los sistemas de } \\
\text { evaluación }\end{array}$ & CPGD \\
$\begin{array}{l}\text { 13. Cumplir el programa / guía de la asignatura } \\
\text { 14. Coordinarse con otros profesores de la asignatura }\end{array}$ & CPGD \\
$\begin{array}{l}\text { 15. Coordinarse con el profesorado de otras asignaturas } \\
\text { 16. Introducir innovaciones que ayuden a mejorar el } \\
\text { proceso de enseñanza-aprendizaje }\end{array}$ & CT \\
\hline $\begin{array}{l}\text { CI: competencia interpersonal; CM: competencia metodológica; CC: competencia } \\
\text { comunicativa; CPGD: competencia de planificación y gestión docente; CT: } \\
\text { competencia de trabajo en equipo; CDI: competencia de innovación docente. }\end{array}$ & \\
&
\end{tabular}

el profesorado. La encuesta se revisó y analizó en grupos de discusión y se validó en una prueba piloto con una muestra de estudiantes voluntarios de los gruposclase del profesorado participante en el proyecto. Valoraron si el contenido de los ítems y su formulación eran comprensibles y adecuados al nivel de los estudiantes. Las sugerencias de profesores y estudiantes se incorporaron a la redacción final de los ítems, que es la que se pasó a todos los estudiantes de las universidades participantes.

La encuesta se organizó en cuatro apartados:

- En el primero se recogía información general sobre el perfil de los participantes (universidad de procedencia, rama de conocimiento, nivel de estudios cursado, curso, sexo y edad).

- En el segundo apartado se preguntaba a los estudiantes «¿cómo crees que debería ser un buen docente?», con preguntas relacionadas con los distintos componentes que definían las competencias docentes identificadas para el profesorado. Se planteaban 16 enunciados que había que valorar en una escala de 1 a 10, teniendo en cuenta que 1 equivalía a «nada importante para ser un buen docente» y 10 a «muy importante para ser un buen docente» (en el caso del profesorado, la escala era de 1 a 4). Los tres primeros ítems de la encuesta de los estudiantes se referían a la competencia interpersonal; los ítems 4, 5, 6 y 7, a la metodológica; los ítems 8 y 9 a la comunicativa; los ítems 10, 11, 12 y 13 a la de planificación y gestión de la docencia; los ítems 14 y 15 a la de trabajo en equipo; y el ítem 16 a la de innovación.

Los ítems que había que valorar estaban directamente relacionados con los elementos que configuran cada competencia, por lo que pudo establecerse la equivalencia mostrada en la tabla 2 :

- El tercer apartado era de campo abierto, donde los estudiantes pudieran indicar las características que consideraban que debería tener un buen docente y que no estaban recogidas en el listado anterior.
Tabla 3. Equivalencia de las competencias valoradas en la encuesta de los estudiantes y las competencias valoradas en la encuesta del profesorado

\begin{tabular}{ll}
\hline $\begin{array}{l}\text { Competencias priorizadas en la encuesta al } \\
\text { estudiantado }\end{array}$ & $\begin{array}{l}\text { Competencia } \\
\text { equivalente }\end{array}$ \\
\hline Que tenga habilidades interpersonales & CI+CC \\
Que utilice metodologías docentes adecuadas & CM \\
Que sea creativo y tenga capacidad de innovar & CDI \\
$\begin{array}{l}\text { Que planifique bien la docencia (contenido, } \\
\text { temporalización, evaluación, coordinación con otros } \\
\text { profesores...) }\end{array}$ & CPGD \\
\hline $\begin{array}{l}\text { CI: competencia interpersonal; CM: competencia metodológica; CC: competencia } \\
\text { comunicativa; CPGD: competencia de planificación y gestión docente; } \\
\text { CT: competencia de trabajo en equipo; CDI: competencia de innovación docente. }\end{array}$
\end{tabular}

- En el cuarto apartado se ofrecía una escala para priorizar las competencias según la importancia que les concedían (1 la considerada como más importante y 4 como la menos importante). La respuesta era sin repetición, cada número podía utilizarse una única vez.

Las competencias consideradas fueron las siguientes:

- Que tenga habilidades interpersonales.

- Que utilice metodologías docentes adecuadas.

- Que sea creativo y tenga capacidad de innovar.

- Que planifique bien la docencia (contenidos, temporalización, evaluación, coordinación con otros profesores...).

Estas competencias pueden asimilarse a las utilizadas en la encuesta de profesorado, tal como se indica en la tabla 3.

Al seleccionar las competencias que deberían priorizarse en la encuesta de estudiantes, se decidió incorporar en una única competencia la interpersonal y la comunicativa, entendiendo que desde el punto de vista del estudiante, la primera puede incluir la segunda. No se tuvo en cuenta en la priorización la competencia de trabajo en equipo, al hacer referencia a aspectos del trabajo dentro del equipo docente $y$, por tanto, al considerar que el alumnado no siempre tiene acceso a esta información. En último lugar se dejó un apartado abierto para la formulación de observaciones y/o sugerencias.

\section{Procedimiento}

Se calculó la muestra mínima requerida por universidad, área de conocimiento y, en la de los estudiantes, por curso según fuera de grado y de máster. Para ambas encuestas se calculó la muestra mínima fijando un error máximo del 5\%, un intervalo de confianza del 95\% y un nivel de homogeneidad del 50\%.

Se guardó la proporcionalidad necesaria de acuerdo con las poblaciones a las que corresponden (muestra aleatoria estratificada con fijación proporcional).

Se corrigió el sesgo muestral de las universidades con una representatividad mayor realizando un remuestreo aleatorio para garantizar la representatividad real de la población de origen, para obtener así una muestra ponderada de las universidades participantes que es la que se utilizó para este estudio. En ambos colec- 
tivos se descartaron las encuestas incompletas, no finalizadas o con errores.

La encuesta del profesorado se difundió a través de los ICE y las unidades de formación, o centros homólogos, de cada universidad participante, por correo electrónico.

Para la difusión de la encuesta a los estudiantes, cada universidad utilizó el medio y vía de difusión que consideró más conveniente.

\section{Análisis de datos}

Para el análisis de los datos cuantitativos se utilizaron técnicas descriptivas univariantes y multivariantes, para mostrar cómo los encuestados valoraron cada una de las competencias, y sus indicadores según sexo, rama de conocimiento y categoría profesional de los encuestados, edad (categorizada) y años de experiencia. El análisis de la varianza de los distintos factores se realizó con un test ANOVA.

Para analizar si las diferencias entre las medias obtenidas para cada categoría eran significativas, se utilizó la prueba de Kruskal-Wallis, debido a que las variables estudiadas no seguían la distribución normal.

Para el análisis de los datos cualitativos correspondientes a las preguntas abiertas se trataron todas las respuestas conjuntamente, se categorizaron siguiendo la propuesta de indicadores para las competencias docentes elaborado en el marco del grupo GIFD y se organizaron atendiendo a si estas hacían referencia a una competencia u otra.

A partir de los datos obtenidos de la encuesta, y después de realizar el proceso de depuración, se aplicaron técnicas de estadística descriptiva e inferencial y también se analizó el contenido de las preguntas abiertas utilizando el programa ATLAS.ti.

\section{Resultados}

\section{Características de la muestra del profesorado}

El total de docentes que respondieron a la encuesta fue de 7.553, que corresponde al 6.73\% del total de PDI de las universidades españolas, en el período muestreado. Una vez eliminadas las no válidas y recalculado el número necesario para mantener la proporcionalidad por universidades, se trabajó con 5.139 encuestas, que corresponde al $68.03 \%$ del total de encuestas recibidas.

Por comunidades autónomas, las que contaron con mayor representación fueron Cataluña (39.86\%), Andalucía (20.38\%), Aragón (8.70\%), Comunidad Valenciana $(8.08 \%)$, Galicia (6.44\%), Región de Murcia (6.27\%), Illes Balears (2.8\%) y Canarias (1.62\%). Las universidades no presenciales representaron un 3.08\% de la muestra final.

Por sexo, se observó una ligera tendencia de las mujeres a valorar más alto que los hombres. Este patrón se repite para cada una de las competencias. La diferencia es estadísticamente significativa para la mayoría de las competencias y los ítems asociados a ellas ( $\mathrm{p}<.05)$, excepto para la competencia de planificación y gestión de la docencia y de trabajo en equipo, que no se observa una diferencia significativa.

Por rama de conocimiento, la distribución fue del $26.1 \%$ de profesores de Ciencias Sociales y Jurídicas, del $22.5 \%$ de Ingeniería y Arquitectura, del 19.4\% de Arte y Humanidades, del 16.6\% de Ciencias de la Salud y del $15.4 \%$ de Ciencias. Se observó una tendencia del profesorado de Ciencias e Ingeniería y Arquitectura a valorar más bajo que el resto la mayoría de competencias $(p<.05)$.

Por categoría profesional, la distribución de respuestas fue del $47.2 \%$ para PDI funcionario (catedráticos y titulares de universidad), del $43.6 \%$ para PDI contratado (profesor asociado, colaborador, contratado doctor o ayudante doctor) y del $9.2 \%$ para PDI con otros tipos de afiliación (profesores visitantes, eméritos, becarios pre doctorales, etc.). No se observaron diferencias significativas en la valoración de las competencias.

Por edad, la media era de 44.91 años, la media de edad de los hombres (46.26 años) era ligeramente superior que la de las mujeres (43.35 años) ( $\mathrm{p}<.01)$.

Se observaron diferencias significativas en relación con los años de experiencia docente $(\mathrm{p}<.05)$, de los que el $46 \%$ correspondía a profesorado con más de 15 años de experiencia.

\section{Características de la muestra de estudiantes}

Las respuestas obtenidas para estudiantes de grado o licenciatura suponen casi el doble de la muestra estimada como necesaria inicialmente (5.629), por lo que se consideró que la muestra global era representativa de la opinión de los estudiantes universitarios de estas universidades.

Por ramas de conocimiento la distribución fue la siguiente: Ciencias Sociales y Jurídicas (33\%), Ingeniería y Arquitectura (30\%), Ciencias de la Salud (16\%), Artes y Humanidades (13\%), Ciencias (8\%).

En la distribución por cursos, el porcentaje de respuestas osciló entre el $25.4 \%$ de primer curso, el $18.8 \%$ de segundo, el $23.6 \%$ de tercer curso y el $22.9 \%$ de cuarto curso. En cursos superiores, posiblemente relacionados con estudiantes de licenciatura o algunos grados con una duración de cinco o más años, el número de respuestas fue considerablemente inferior: el $8.6 \%$ para estudiantes de quinto curso y el $0.7 \%$ para los de sexto curso.

La distribución de respuestas atendiendo al sexo y a la edad el 59.6\% correspondían a mujeres y el 40.4\% a hombres, con una media de edad de 22.9 años y una desviación estándar de 6.6. Esta distribución reflejó la realidad del momento en que se hizo el estudio ya que, según datos del INE, el 55.3\% de los estudiantes aprobados en las pruebas de acceso a la universidad, en la convocatoria de 2012, fueron mujeres.

Comparando las respuestas por ramas de conocimiento, el número de hombres fue superior al de mujeres en Ingeniería y Arquitectura; la menor diferencia entre ambos se encontró en Ciencias (45.5\% 
hombres y $54.5 \%$ mujeres) y la mayor diferencia fue en Ciencias de la Salud (76.3\% mujeres frente a $23.7 \%$ hombres), seguida de Ciencias Sociales y Jurídicas (72.7\% vs. 27.3\%).

Para los estudiantes de máster, las respuestas obtenidas (1.700) suponen solo el $37.7 \%$ de la muestra estimada como necesaria inicialmente (4.505) para que los datos sean representativos. No obstante, se realizó igualmente el análisis de tendencias observadas. Solo dos universidades (UIB, UPC) superaron la muestra estimada inicialmente y, en consecuencia, el análisis de los datos individuales de estas universidades sí era representativo de la opinión de sus estudiantes de máster. De todos modos, igual que con los estudiantes de grado, los datos se trataron a escala global, por lo que la distribución de la muestra era adecuada. Además, los datos se trataron de manera descriptiva (no inferencial).

La distribución de las respuestas de máster por ramas de conocimiento sigue un patrón similar al obtenido en las encuestas de grado, pues prácticamente dos terceras partes de los estudiantes que participaron en el estudio correspondían a las mismas ramas de conocimiento: Ciencias Sociales y Jurídicas (30\%) y de Ingeniería y Arquitectura (30\%). En el resto se produce una ligera variación en el orden, pues el porcentaje de respuestas de los estudiantes de Arte y Humanidades (18\%) supera al de los estudiantes de Ciencias de la Salud (11\%) y de los de Ciencias que, de nuevo, son quienes menos expresaron su opinión sobre el tema (11\%).

Atendiendo al sexo y a la edad de los estudiantes que respondieron a las encuestas, también entre los estudiantes de máster, la mayoría (55.5\%) fueron mujeres frente al $44.5 \%$ de hombres, con una media de edad de 29.1 años y una desviación estándar de 7.97. El 25\% por ciento de los estudiantes tenían menos de 24 años, y otro $25 \%$ superaba los 32 años.

\section{Discusión}

\section{Valoración de la competencia de innovación docente por parte del profesorado}

De la valoración media que hicieron los docentes en relación con la importancia de las competencias en el ejercicio profesional, la de innovación docente se situó en quinta posición con un valor medio de 3.33 sobre una escala de 1 a 4 (la más valorada obtuvo un valor de 3.64 y la menos de 3.27). A pesar de su posición, el valor asignado permite afirmar que la consideraban como «bastante importante (50.57\%) o muy importante (40.63\%)». Estos resultados son comparables a los encontrados por Triadó et al. (2014) y Torra et al. (2012), que describen que el profesorado de las universidades catalanas priorizaba la importancia de esta competencia en último lugar, en relación con las otras competencias, a pesar de que en valores absolutos estaba mayoritariamente bien y muy bien valorada. Estos resultados sugieren que el profesorado tiene una tarea pendiente en este campo y que no concibe que dentro de su actuación docente deba contemplar la introducción de innovaciones docentes como parte de su proceso de mejora de la calidad docente.

De entre los elementos que componen la competencia de innovación, el más importante según los encuestados era «introducir innovaciones que tengan como objetivo una mejora del proceso de enseñanzaaprendizaje (CDI5)». El 45.67\% de los encuestados consideraba que este elemento es «muy importante»y el $46.22 \%$ consideraba que es «bastante importante». En cambio, lo que consideraban menos importante era «participar activamente en proyectos y experiencias de innovación docente (CDI6)», en la que solo un $31.56 \%$ consideraba como «muy importante» y un $21.17 \%$ de los encuestados consideraba «poco» o «nada» importante. En la tabla 4, se muestran las medias finales para cada elemento y las desviaciones estándar (DE), teniendo en cuenta los valores globales, ordenados de mayor a menor importancia.

Por categoría profesional, los colectivos que más valoraron la competencia de innovación fueron los profesores «visitantes» (con una valoración media de 3.5), seguidos de los profesores «colaboradores» (con una valoración media de 3.45) y los profesores «contratados doctor» (con una valoración media de 3.49). Y los que menos, los profesores «catedráticos» (con una valoración media de 3.13) y los profesores «titulares» (con una valoración media de 3.28). Cabe destacar que, en general, estos últimos tendieron a valorar más bajo las diferentes competencias y los elementos que las conforman.

Sobre todo, destaca la poca importancia que dieron los profesores «catedráticos» respecto del resto a «participar activamente en proyectos y experiencias de

Tabla 4. Puntuación media y desviación estándar de los indicadores de la competencia de innovación

\begin{tabular}{|c|c|c|}
\hline Ítem & Media & $\mathrm{DE}$ \\
\hline CDI5. Introducir innovaciones que tengan como objetivo una mejora del proceso de enseñanza-aprendizaje & 3.36 & .664 \\
\hline $\begin{array}{l}\text { CDI1. Analizar el contexto de enseñanza-aprendizaje para identificar las necesidades de mejora y aplicar estrategias y/o } \\
\text { recursos innovadores }\end{array}$ & 3.28 & .672 \\
\hline $\begin{array}{l}\text { CDI2. Reflexionar e investigar sobre los procesos de enseñanza-aprendizaje para buscar nuevas estrategias que permitan } \\
\text { mejorarlos }\end{array}$ & 3.25 & .700 \\
\hline CDI4. Adaptar las innovaciones a las características y particularidades de cada contexto & 3.25 & .695 \\
\hline $\begin{array}{l}\text { CDI7. Evaluar y transferir resultados y experiencias de innovación al propio contexto de enseñanza-aprendizaje orientadas } \\
\text { a la mejora de la calidad docente }\end{array}$ & 3.17 & .745 \\
\hline CDI3. Definir un objetivo preciso de la innovación que se pretende llevar a cabo & 3.13 & .738 \\
\hline CDI6. Participar activamente en proyectos y experiencias de innovación docente & 3.07 & .791 \\
\hline
\end{tabular}


innovación docente (CDI6)». También los profesores «eméritos» daban menos importancia que el resto a este aspecto. En cambio, prácticamente había coincidencia en señalar como más importante para la función docente el «introducir innovaciones que tengan como objetivo una mejora del proceso enseñanza-aprendizaje (CDI5)», excepto el perfil docente «visitantes» que consideraron más importante «analizar el contexto de enseñanza-aprendizaje para identificar las necesidades de mejora y aplicar estrategias y/o recursos innovadores (CDI1)» $\mathrm{y}$ «reflexionar e investigar sobre los procesos de enseñanza-aprendizaje para buscar nuevas estrategias que permitan mejorarlos CDI2)». En el caso de los eméritos, también valoraron mejor que el resto «definir un objetivo preciso de innovación que se pretende llevar a cabo».

Por años de experiencia docente, la mejor valoración fue la del profesorado entre 3 años y 10 de experiencia docente. Concretamente, valoraron mejor los elementos «participar activamente en proyectos y experiencias de innovación» $\mathrm{y}$ «evaluar y transferir resultados y experiencias de innovación al propio contexto de aprendizaje para la mejora de la calidad docente».

Respecto a la variable edad, los datos no presentan diferencias significativas.

Por rama de conocimiento, el profesorado que más valoró la competencia de innovación fue el del ámbito de Ciencias de la Salud (3.47), seguido del profesorado de Ciencias Sociales y Jurídicas (3.36) y de Arte y Humanidades (3.33). Asimismo, se observó la tendencia del profesorado de Ciencias e Ingeniería y Arquitectura a valorar más bajo que el resto $(\mathrm{p}<.05)$. Sin embargo, todos coincidían en señalar como más importante para la función docente el hecho de «introducir innovaciones que tengan como objetivo una mejora del proceso enseñanza-aprendizaje (CDI5)» y también coincidieron en señalar como los elementos menos importantes de esta competencia el «participar activamente en proyectos y experiencias de innovación docente (CDI6)» $\mathrm{y}$ «definir un objetivo preciso de la innovación que se pretende llevar a cabo (CDI3)».

Por sexos, se observó una tendencia de las mujeres a valorar más alto que los hombres. La diferencia es estadísticamente significativa para todas las competencias e ítems asociados a ellas $(\mathrm{p}<.05)$. A pesar de estas diferencias en la valoración, hubo coincidencia entre hombres y mujeres a la hora de señalar los elementos más importantes de cada competencia.

\section{Priorización}

La última parte del cuestionario tenía como objetivo medir la importancia relativa que los encuestados daban a cada una de las competencias, asignando un 1 a la más importante y un 6 a la menos importante. La competencia de innovación docente quedó clasificada en última posición entre las seis competencias. Recordemos que en la escala de la valoración de su importancia relativa estaba en quinta posición con un valor de 3.33 en una escala de 1 a 4 .

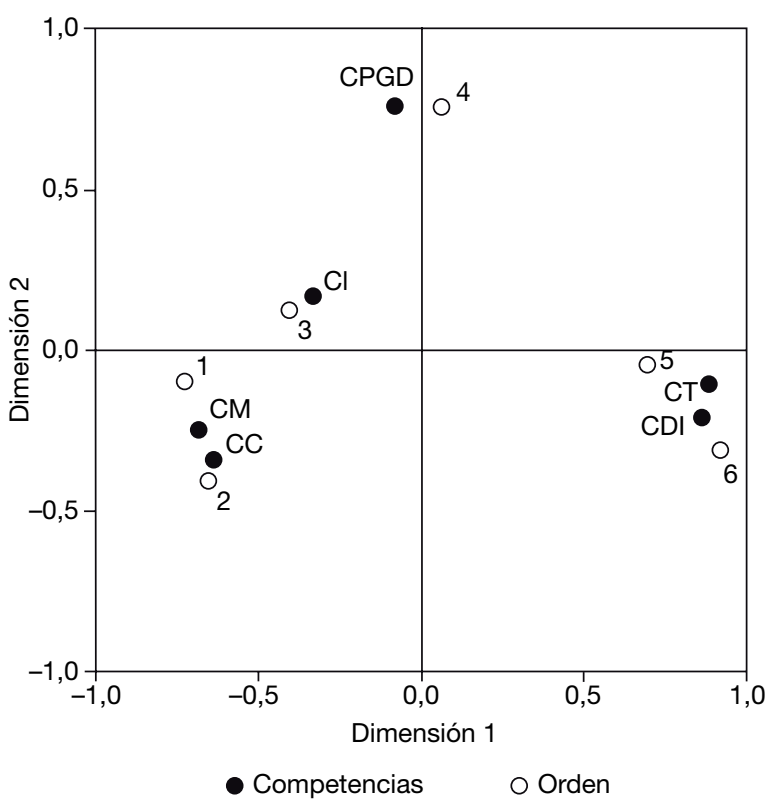

Figura 1. Análisis factorial de correspondencia de las valoraciones de las competencias docentes por parte del profesorado Cl: competencia interpersonal; CM: competencia metodológica; CC: competencia comunicativa; CPGD: competencia de planificación y gestión docente; CT: competencia de trabajo en equipo; CDI: competencia de innovación docente.

Dado que todas las competencias se puntuaron con un elevado nivel de importancia, la priorización no permitió discriminarlas claramente. Para analizar los datos resultantes de la priorización se utilizó una técnica estadística multivariante, el análisis factorial de correspondencias (AFC). El resultado de este análisis permitió agrupar los datos en dos dimensiones o factores, que retienen el 96\% de la información de los datos. El primer factor (Dimensión 1) explica un $86 \%$ de la información y contrapone las competencias metodológica, comunicativa e interpersonal a las competencias de innovación y trabajo en equipo. La competencia de planificación y gestión de la docencia define el segundo factor (Dimensión 2) que explica un 10\% de la información. En la figura 1 se puede observar la proximidad de cada competencia al lugar que ocupa en la priorización. Así, la competencia metodológica (CM) aparece en primera posición, seguida de la comunicativa (CC) y de la interpersonal (CI) en tercera posición, mientras que las competencias de innovación (CDI) y de trabajo en equipo (CT) se sitúan entre el quinto y sexto lugar. En la figura 1 se observa que la de planificación y gestión docente (CGPD) aparece en cuarto lugar, pero definiendo por sí misma una dimensión perpendicular (poco relacionada) a la formada por el resto de componentes; de esta manera se identifica qué competencias se perciben como parecidas y cuáles de ellas se contraponen.

\section{Resultados cualitativos de la encuesta del profesorado}

La encuesta incluía varias preguntas abiertas en las que se pedía al profesorado, si lo creía oportuno, que propusiera otros elementos para la definición de cada una de las competencias. 
La pregunta 33 de la encuesta pedía que, una vez valoradas las seis competencias propuestas, añadieran, de manera opcional, otras competencias que consideraran igualmente relevantes.

Para realizar el análisis de los datos se trataron todas las respuestas conjuntamente, categorizándolas siguiendo la propuesta de indicadores para las competencias docentes y se organizaron atendiendo a si esta categoría hacía referencia a una competencia u otra.

La categorización para la competencia de innovación docente se realizó sobre la propuesta de nuevos elementos para su definición, a factores externos que dificultan el desarrollo de esta competencia y al grado de acuerdo de los elementos que la conforman.

El número de aportaciones para esta competencia fue del $10.05 \%$. La mayoría de ellas (58.46\%) incidía en la necesidad de que la innovación no fuera un objetivo en sí mismo, sino que se orientara a mejorar la calidad docente. Por tanto, harían referencia al elemento «introducir innovaciones que tengan como objetivo una mejora del proceso de enseñanza-aprendizaje». En este sentido, el profesorado también mencionaba «el uso de recursos TIC para la mejora docente y, en general, «el aplicar la creatividad en la docencia».

El $23.08 \%$ de las aportaciones mencionaba el elemento «evaluar y transferir resultados y experiencias de innovación al propio contexto de enseñanzaaprendizaje orientados a la mejora de la calidad docente». Aquí se incluyen aspectos como «experimentar innovaciones directamente en el aula», «transferir los resultados de la investigación a la docencia»y, sobre todo, «evaluar los resultados de la innovación». El énfasis en la evaluación de los resultados estaría relacionado también con el objetivo de mejorar el proceso de enseñanza-aprendizaje, que, en general, el profesorado considera importante no perder de vista.

El $6.15 \%$ de las aportaciones tenía que ver con «reflexionar e investigar sobre los procesos de enseñanza-aprendizaje para buscar nuevas estrategias que permitan mejorarlos». Concretamente, el profesorado proponía investigar sobre experiencias de innovación de otros centros y países y/o participar en foros y jornadas de innovación docente como medio para conocer nuevas estrategias.

Otros elementos propuestos fueron: «incorporar innovaciones en el contenido de la materia» (9.23\%), «aplicar estrategias y recursos innovadores, no solo en el aula, sino también en la gestión o en la tutoría» (1.54\%) y, por último, «disponer de formación pedagógica» (1.54\%).

\section{Valoración de la competencia de innovación docente por parte de los estudiantes}

Las puntuaciones que otorgó el alumnado de grado a los diferentes ítems de la encuesta, en una escala de 1 al 10, estaban entre sobresaliente (9.46) y notable alto (7.55), apenas existía una diferencia de 1.91 puntos en el conjunto de ítems valorados. En cambio, las puntuaciones en el máster se situaron entre los 8.12 y 9.31 .
El ítem relacionado con la competencia de innovación «introducir innovaciones que ayuden a mejorar el proceso de enseñanza-aprendizaje» los estudiantes de grado la valoraron con una puntuación media de 8.27 ( \pm 1.7$)$, mientras que los de máster le dieron una puntuación de 8.40 ( \pm 1.627$)$, lo que la situaba en el duodécimo lugar de los dieciséis ítems totales que había que valorar, en los dos colectivos.

La puesta en marcha de innovaciones para mejorar el proceso de enseñanza-aprendizaje es más importante para los estudiantes de Ciencias Sociales y Jurídicas y para los de Ciencias de la Salud (media sobre 8.5). Los que concedían menor importancia a esta competencia para ser un buen docente son los que estudiaban grados de las ramas de Ingeniería, Arquitectura o Ciencias (media en torno a 8). Los estudiantes de Arte y Humanidades concedían una importancia media a este hecho. La prueba Kruskal-Wallis de muestras independientes nos permite afirmar que, efectivamente, hay diferencias en las opiniones de los estudiantes según su rama de conocimiento en todas ellas, con un nivel de significación de $\mathrm{p}<.05$.

Nos parece interesante resaltar que tanto para los estudiantes de grado, como para los de máster, este ítem ocupaba el duodécimo lugar de los dieciséis aspectos valorados, con lo que comparativamente la valoraban un poco mejor que el profesorado, que la situó entre las menos importantes para el desempeño de su función docente, aunque la puntuación dada fue alta, por lo que podía considerarse dentro del rango de «importante».

Comparando las respuestas por cursos, los estudiantes de primer curso valoraron más alto que los de los cursos superiores el ítem relacionado con la «introducción de innovaciones en la docencia». $(\mathrm{p}<.05)$

En relación con el sexo, para todos los ítems de la encuesta las puntuaciones medias fueron significativamente mayores en las respuestas de las mujeres que en los hombres $(\mathrm{p}<.05)$.

En la priorización sí hubo coincidencia entre los resultados encontrados en la encuesta del profesorado y la de los estudiantes. Los dos colectivos priorizaron la competencia de innovación docente en último lugar de su escala de valoración (entre 1 menos importante, a 4 más importante), considerándola como la menos importante, y entre estudiantes de grado $(2.17, \pm 1.11)$ y de máster $(2.22, \pm 1.11)$ no se encontró ninguna diferencia significativa. Estos resultados, en el caso de los estudiantes, pueden llevar a pensar que no tienen la información contextual necesaria para interpretar el significado de cada competencia docente, o no saben valorar la importancia que la innovación pueda tener para la calidad docente. Esta premisa coincide con los estudios más pormenorizados llevados a cabo con los estudiantes de la Universidad de Barcelona (Sayós, Pagés, Amador \& Jorba, 2014). Más problemático es explicar por qué los profesores la priorizaban en último lugar. Debería ampliarse el estudio en este aspecto para tratar de encontrar el porqué de esta situación. 


\section{Resultados cualitativos de la encuesta de los estudiantes}

En las cuestiones abiertas, los estudiantes hicieron aportaciones, muchas de las cuales hacían referencia a las seis competencias. Una vez analizadas para grado y máster, al no observarse diferencias significativas, se consideró oportuno hacer una discusión global de ambos colectivos.

Los 16 ítems de la encuesta se conceptualizaron en distintos códigos de análisis, los cuales se correspondían con indicadores de cada competencia. La mayoría de las citas (quotations) hacían referencia a las seis competencias docentes establecidas. De las respuestas que no se correspondían con las competencias definidas, algunas se relacionaban con aspectos como el conocimiento de la materia y la experiencia profesional del docente, por lo que se agruparon bajo el epígrafe de competencia técnico-científica. El resto se integró bajo el epígrafe «Otras», ya que acogía aspectos muy diversos, como el cumplimiento de las obligaciones docentes, la libertad docente o el nivel de exigencia.

La competencia de innovación docente la relacionaron con la formación permanente del profesorado, su creatividad, su excelencia investigadora y su capacidad de adaptarse a entornos cambiantes.

Su opinión se centraba en que los docentes habían de participar en investigaciones y realizar formación continua, con el objetivo de actualizarse y a partir de aquí, introducir innovaciones en el aula que mejoraran el proceso de enseñanza y aprendizaje. En definitiva, ponían énfasis en la formación docente a lo largo de toda su trayectoria profesional.

Otras veces mencionaban que los docentes aplicaban metodologías y contenidos antiguos, que no se acababan de adaptar a la actualidad y ofrecían material docente desfasado.

En este sentido, las aportaciones ponían de manifiesto que hay profesorado que intentaba introducir «innovaciones», pero a veces estas podían entorpecer el proceso de enseñanza-aprendizaje, más que mejorarlo. Indicaban que hay que tener cuidado con «intentar innovar», ya que se debe entender la innovación enfocada a la mejora.

En este sentido, el avance hacia la convergencia europea significa una posibilidad de innovación para los títulos universitarios y, tal como recogían los estudiantes, esta competencia está estrechamente relacionada con el pensamiento creativo (Stenberg \& Lubart, 1997) y con el pensamiento divergente (De Bono, 1986; Guilford, 1950). Las reflexiones realizadas por los estudiantes pueden ser de gran utilidad para completar la definición de las competencias docentes y para identificar los indicadores que permitirán observar su nivel de progreso (Sayós et al., 2014).

\section{Niveles competenciales}

Partiendo de las competencias identificadas y definidas, y de la propuesta de dimensiones e indicadores para cada una de ellas, se consensuaron tres niveles progresivos de desarrollo competencial: novel o inicial, sénior y experto. Una vez fijados estos tres niveles, se analizaron los indicadores de cada una de las dimensiones de las seis competencias docentes, y se clasificaron según su grado de complejidad, para vincularlos a un nivel de desarrollo competencial. El proceso se completó con la identificación de las evidencias que pudieran aportar información para la evaluación de los distintos niveles de progreso del desarrollo competencial del profesorado. En concreto, para la competencia de innovación, los criterios que se aplicaron para clasificar por niveles los indicadores de sus dimensiones son (Pagés et al., 2013):

Primer nivel: participar en cursos de formación y documentarse para poder llevar a cabo las acciones de mejora. Analizar el proceso de enseñanza-aprendizaje para identificar puntos débiles y proponer soluciones de mejora;

Segundo nivel: planificar acciones de mejora e integrarlas en su asignatura teniendo en cuenta las características del contexto. Formar parte de un equipo docente y poner en práctica innovaciones acordadas por el equipo.

Tercer nivel: evaluar las innovaciones llevadas a cabo; impulsar la acción de equipos docentes. Compartir acciones de innovación con otros grupos y transferirlas a otros contextos.

Las pruebas o evidencias para determinar el grado de desarrollo alcanzado para cada competencia pueden obtenerse de instrumentos (documentos, grabaciones, informes, entrevistas, certificaciones, testimonios, observaciones, etc.) que pongan de manifiesto la actuación docente en situaciones específicas, como puede ser en el diseño de una asignatura, en la aplicación en el aula de los procesos de enseñanza-aprendizaje, en los materiales que se utilizan, en la participación en convocatorias de mejora e innovación docente, asistencia a congresos o publicaciones docentes (Ruiz Bueno, Mas Torelló, Tejada Fernández \& Navío Gómez, 2008; Tejada Fernández, 2009; Mas \& Tejada, 2013). El objetivo es facilitar el análisis del impacto de las acciones orientadas al desarrollo de la competencia.

En relación con este punto es preciso comentar que todas las universidades participantes en el estudio ofrecen cursos, tanto de formación inicial como permanente, relacionados con la competencia de innovación docente, $y$, aunque también manifiestan que es una de las competencias menos trabajadas, el profesorado que ha recibido formación referente a esta competencia consideró que la formación recibida sobre «mejoras en el proceso de enseñanza-aprendizaje e introducción de nuevas metodologías docentes y su evaluación» era útil o muy útil para la mejora de su práctica docente. Estas acciones estarían en la línea de lo que proponen Zabalza (2013) y Rodríguez Conde et al. (2014) en referencia a la formación docente y al desarrollo de la competencia de innovación educativa como elementos clave de la calidad institucional. 
Como es sabido, la universidad vive en un contexto de cambio y los docentes han de estar preparados para hacer frente a los nuevos retos, como agente de impacto en el cambio orientado a la mejora. A través de una formación continua atenta a las necesidades docentes, enfocada al buen funcionamiento de su programa docente, que combinara recursos técnicos y didácticos y que lo hiciera competente para su función docente (Salinas, 2004; Torra et al., 2013).

El estudio sobre las competencias, como la de innovación docente, ha puesto de manifiesto la necesidad de explicitar cómo deben encaminarse las acciones para el desarrollo de esta competencia, de introducir mejoras en los procesos e instrumentos que ayuden a adquirirla por parte del profesorado y de cómo estos pueden aplicar su conocimiento en el aula.

\section{Conclusiones}

Tanto el profesorado como el alumnado universitario participante en el estudio configuran una muestra representativa de las universidades españolas, para los perfiles definidos para cada colectivo.

Desde la perspectiva docente, se considera la competencia de innovación como una de las menos importantes de las seis definidas.

De acuerdo con los resultados obtenidos entre los estudiantes, las puntuaciones medias de todas las preguntas son altas, lo que indica la importancia que el alumnado otorga a las competencias que ha de tener un buen docente.

Cabe destacar las diferencias significativas en las opiniones de los estudiantes sobre la competencia de innovación docente, según su rama de conocimiento de procedencia. Asimismo, se observa que esta competencia va teniendo menor importancia para el alumnado, según va avanzando en su formación universitaria.

Tanto para los estudiantes de grado como para los de máster «introducir innovaciones para mejorar el proceso de enseñanza-aprendizaje» ocupa el duodécimo lugar de los dieciséis aspectos valorados, aunque le otorgan una puntuación alta.

Al relacionar los resultados de la priorización de los estudiantes con la que realizaron los profesores, ambos coinciden en valorar la competencia de innovación como una de las menos importantes.

La poca importancia concedida a la competencia de innovación es un dato que hay que considerar para emprender acciones de seguimiento de la calidad y la incorporación de acciones de mejora en este campo.

\section{Referencias}

Abadía, A.R., Bueno, C., Ubieto-Artur, M.I., Márquez, D., Sabaté, S., Jorba, H. \& Pagès, T. (2015). Competencias del buen docente universitario. Opinión de los estudiantes. Revista de Docencia Universitaria, 13(2), 363-390.
Bain, K. (2006). Lo que hacen los mejores profesores universitarios. Experiencias y propuestas para la docencia universitaria. Valencia: Publicaciones Universidad de Valencia.

Barnett, R. (2011). Being a University. Nueva York: Routledge.

Biggs, J. B. (2003). Teaching for Quality Learning at University. Oxford: Open University Press.

De Bono, E. (1986). El pensamiento lateral. Barcelona, España: Paidós.

Euler, D. (2015). Mejorar las competencias docentes del profesorado universitario es necesario, pero la innovación sostenible requiere algo más! Educar, 51(1), 149-165.

Gairín, J. (2011) Prólogo. En M. I. Vázquez, Procesos de calidad y autoevaluación en centros educativos. ¿Dos cara de una misma moneda? (pp. 9-19). Barcelona: DaVinci.

González, J. \& Wagenaar, R (Eds.) (2003). Tuning Educational Structures in Europe. Informe final fase I. Bilbao: Publicaciones de la Universidad de Deusto. Recuperado de, http://docplayer.es/8488102-Tuning-educational-structures-in-europe.html

González, J. \& Wagenaar, R (Eds.) (2006). Tuning Educational Structures in Europe II. Informe final. La contribución de las universidades al Proceso de Bolonia. Bilbao: Publicaciones de la Universidad de Deusto. Recuperado de, http://www.deusto-publicaciones.es/ deusto/pdfs/tuning/tuning04.pdf

Guilford, J. P. (1950). Creativity. The American Psychologist, 5(9), 444-454.

Llena, A. \& Parcerisa, A. (2008). La acción socioeducativa en medio abierto. Barcelona: Graó.

Mas, O. \& Tejada, J. (2013). Funciones y competencias en la docencia universitaria. Barcelona: Síntesis.

Pagés, T., Sayós, R., Triadó, X., Hernández, C., Solà, P. \& Baños, E. (2013). Indicadores para la evaluación del perfil competencial docente del profesorado universitario. En T. Ramiro-Sánchez y M. T. Ramiro (Comps.), FECIES 2013. (pp. 100-105). Granada: Asociación Española de Psicología Conductual (AEPC).

Pagés, T. (Coord.). (2014). Propuesta de un marco de referencia competencial del profesorado universitario y adecuación de los planes de formación basados en competencias docentes. Memoria final. Red Estatal de Docencia Universitaria. Disponible en:

http://red-u.org/wp-content/uploads/propias/MEMORIA_PROYECTO_REDU2012-Teresa_Pages.pdf

Parcerisa, A., Bascos, S., Calafell, M., Comas, M. \& Noguera, I. (2011). Innovació en educació. De la idea a l'acció. Procés participatiu cap a l'INNED 2. Revista d'Innovació i Recerca en Educació (REIRE), 4(2), 82-95.

Rodríguez Conde, M. J., Olmos Migueláñez, S., Ortega Mohedano, F., Torrijos Fincias, P. \& Hernández Garzón, S. (2014). Evaluación, formación e innovación docente: respuesta a la mejora de calidad universitaria. Revista CIDUI, 2014. 1-17. Recuperado de, www. cidui.org/revistacidui

Rodríguez-Gómez, D. \& Gairín Sallán, J. (2015). Innovación, aprendizaje organizativo y gestión del cono- 
cimiento en las instituciones educativas. Educación, XXIV(46), 73-90.

Ruiz Bueno, C., Mas Torelló, Ó., Tejada Fernández, J. \& Navío Gámez, A. (2008). Funciones y escenarios de actuación del profesor universitario: Apuntes para la definición del perfil basado en competencias. Revista de la educación superior, 37(146), 115-132.

Salinas, J. (2004). Innovación docente y uso de las TIC en la enseñanza universitaria. Revista universidad y sociedad del conocimiento, 1(1), 1-16.

Sayós, R. Pagés, T., Amador, J.A. \& Jorba, H. (2014). Ser buen docente ¿Qué opinan los estudiantes de la Universidad de Barcelona? Revista Iberoamericana de Psicología y Salud, 5(2), 135-149.

Stenberg. R. J. y Lubart, T. I. (1997). La creatividad en una cultura conformista. Un desafío de las masas. Barcelona. España: Paidós.

Tejada Fernández, J. (2009). Competencias docentes. Profesorado. Revista de currículum y formación del profesorado. 13(2), 1-15.

Torra, I. (Coord.) (2011): Identificación, desarrollo y evaluación de competencias docentes en la aplicación de planes de formación dirigidos a profesorado universitario. Memoria Final: Programa de estudios y análisis destinado a la mejora de la calidad de la enseñanza superior y de la actividad del profesorado universitario. Ministerio de Educación, Gobierno de España. Disponible en: http://138.4.83.162/mec/ayudas/reposi torio/ $20110930130115 \mathrm{Me}$ moria\% 20 cient\%C3\%ADfica\%20\%28EA2010-0099\%29.pdf

Torra, I., Del Corral, I., Pérez, M.J., Triadó, X., Pagès, T., Valderrama, E., Marques, M.D., Sabaté, S., Solà, P., Hernández, C., Sangrà, A., Guàrdia, L., Estebanell, M., Patiño, J., González, A-P., Fandos, M., Ruiz, N., Iglesias, C. \& Tena, A. 2012. Identificación de competencias docentes que orienten el desarrollo de planes de formación dirigidos a profesorado universitario. Revista de Docencia Universitaria. 10(2), 21-56.

Torra, I., Márquez, M.D., Pagès, T., Solà, P., García, R., Molina, F., González, A. \& Sangrà, A. 2013. Retos institucionales de la formación del profesorado universitario. Revista de Docencia Universitaria, 11(1), 285-309.

Triadó, X., Estebanell, M., Márquez, M.D. \& Del Corral, I. 2014. Identificación del perfil competencial docente en educación superior. Evidencias para la elaboración de programas de formación continua del profesorado universitario. Revista Española de Pedagogía, año LXXII, 257, 51-72.

Zabalza, M.A. 2013. La formación del profesorado universitario. Revista de Docencia Universitaria, 11(3), 11-14.

La innovació com a competència docent a la Universitat: innovació orientada a la millora de l'aprenentatge

Resum. En aquest treball valorem la competència d'innovació docent del professorat universitari com a mitjà per a millorar la qualitat de la docència $i$ aconseguir un millor aprenentatge dels estudiants. Aquesta competència és una de les sis definides com a rellevants, $i$ considerada com una competència significativa pel Grup Interuniversitari de Formació Docent (GIFD), i pel grup d'experts externs. La validació d'aquestes competències es va dur a terme a partir de les dades recollides en enquestes adreçades al professorat $i$ als estudiants de les universitats espanyoles. La doble visió proporcionada pels professors i els estudiants va servir per a redefinir millor les dimensions de cada competència $i$ els indicadors corresponents a cada dimensió. Aquests indicadors es van organitzar per nivells, i es van proposar evidències per poder valorar el nivell competencial del professorat. Aquesta valoració pot ajudar a promoure la millora professional del professorat a través de programes de formació docent basats en competències, redundant en una millora de la seva actuació a l'aula $i$, per tant, de l'aprenentatge dels estudiants. Pot servir, a més, com a sistema de suport per a avaluar la qualitat de la docència en els processos d'acreditació del professorat de les agències de qualitat universitària, autonòmica i estatal.

Paraules clau: competències docents; innovació docent; desenvolupament competencial; formació professorat universitari 
